\title{
Detección de Leishmania spp. (Trypanosomatidae) e identificación de ingestas sanguíneas en flebotomíneos de un nuevo foco de leishmaniasis en el Caribe colombiano.
}

\section{Detection of Leishmania spp. (Trypanosomatidae) and bloodmeal identification in sandflies from a new focus of leishmaniasis in the Colombian Caribbean.}

\author{
Maira Alemán-Santos ${ }^{1}$, Lina Martínez-Pérez ${ }^{1}$, Matilde Rivero-Rodríguez ${ }^{1} \mathcal{D}$, Luis Cortes- Alemán ${ }^{2}$, $^{2}$ \\ Alveiro Pérez-Doria ${ }^{1} \mathcal{B}$, Eduar Bejarano-Martínez ${ }^{1}$ \\ Universidad de Sucre, Sincelejo, Colombia. \\ 2 Secretaria de Salud Departamental de Bolívar, Colombia.
}

*Dirigir correspondencia a: mairaaleman44@gmail.com

\begin{abstract}
RESUMEN
RESUMEN
Introducción: Aunque la leishmaniasis visceral (LV) es endémica en el Caribe colombiano, en los últimos años se ha observado un incremento en su área de distribución, con el registro de casos en nuevas

Proceso Editorial

Recibido: 281220

Aceptado: 160621

Publicado: 241121 localidades. Objetivos: En este estudio se caracterizaron los flebotomíneos, parásitos del género Leishmania y algunos vertebrados domésticos asociados al primer caso humano de LV en la vereda Toro, San Juan Nepomuceno, Bolívar, Colombia. Metodología: Los insectos fueron sometidos a extracción, amplificación y secuenciación de ADN para establecer si estaban infectados con Leishmania spp. e identificar sus ingestas sanguíneas. Adicionalmente, en los caninos se determinaron los títulos de anticuerpos anti-Leishmania mediante la técnica de inmunofluorescencia indirecta. Resultados: En total se recolectaron 2178 flebotomíneos, el 99,6\% de los cuales fue identificado como Lutzomyia evansi. Los parásitos Leishmania infantum y Leishmania braziliensis fueron detectados en esta especie, con una frecuencia mínima de infección de 0,003\% (3/1070) y 0,0009\% (1/1070), respectivamente. El 16,73\% de las hembras de Lu. evansi se encontraron alimentadas de Homo sapiens sapiens, el 16,32\% de Capra hircus , el $12,45 \%$ de Sus scrofa domesticus, el $11,63 \%$ de Bos indicus y el 9,79\% de Canis familiaris. Uno de ocho caninos serológicamente evaluados fue positivo para leishmaniasis canina. Conclusión: El hallazgo en Lu. evansi de ingestas de sangre mixtas de humanos y caninos, evidencia el vínculo epidemiológico entre las Lutzomyia infectados con el parásito, los potenciales reservorios y la población humana, lo que explicaría la aparición del primer caso de LV en esta localidad del Caribe colombiano.

Palabras clave: Colombia; Insectos Vectores; Parásitos; Psychodidae; Trypanosomatida .
\end{abstract}

DOI 10.17081/innosa.142

(c) Copyright 2021.

Alemán $\mathrm{M}^{1}$ et al.

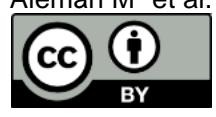

\begin{abstract}
Background: Although the visceral leishmaniasis $(\mathrm{VL})$ is an endemic disease in the Colombian Caribbean, an increase in its distribution area has been observed in the last years, including reports of cases in new locations. Objetives: In this study, we characterized sandflies, parasites from the Leishmania genus, and some domestic vertebrates associated with the first case of VL in the rural settlement of Toro, San Juan Nepomuceno, Bolívar, Colombia. Methodology: The sandflies were subjected to DNA extraction, amplification, and sequencing for detection of Leishmania spp. infection, and identification of bloodmeals. Additionally, we determined anti-Leishmania antibody titres in canines by the indirect immunofluorescence assay technique. Results: A total of 2178 sandflies were collected, of which $99.6 \%$ were identified as Lutzomyia evansi. Leishmania infantum and Leishmania braziliensis were the parasites detected in this sandfly species, with a minimum infection rate of $0.003 \%(3 / 1070)$, and $0.0009 \%(1 / 1070)$, respectively. Regarding bloodmeals, $16.73 \%$ of the Lu. evansi females had fed on Homo sapiens sapiens, $16,32 \%$ on Capra hircus, $12.45 \%$ on Sus scrofa domesticus, $11.63 \%$ on Bos indicus, and $9.79 \%$ on Canis familiaris. One of the serologically assessed canines was positive for canine leishmaniasis. Conclusion: The detection of mixed bloodmeals from humans and canines in Lu. evansi evidences the epidemiological link among sandflies infected with the parasite, potential reservoirs, and human population. This would explain the appearance of the first VL case in this locality of the Colombian Caribbean.

Keywords: Colombia; Insect Vectors; Parasites; Psychodidae; Trypanosomatida
\end{abstract}

Cómo citar: Alemán M, Martínez L, Rivero M, Cortes L, Pérez Alveiro, Bejarano E. Detección de Leishmania spp. (Trypanosomatidae) e identificación de ingestas sanguíneas en flebotomíneos de un nuevo foco de leishmaniasis en el Caribe colombiano. Ciencia e Innovación en Salud. 2021. e142: 311-324 DOI 10.17081/innosa.142

Cite this dataset: Innovación en Salud, Ciencia e (2021), "Detection of Leishmania spp. (Trypanosomatidae) and bloodmeal identification in sandflies from a new focus of leishmaniasis in the Colombian Caribbean", Mendeley Data, V1, doi: 


\section{INTRODUCCIÓN}

En Colombia, la leishmaniasis visceral (LV) es una zoonosis que presenta un carácter endémico y epidémico, los focos más importantes de la enfermedad están distribuidos en el margen del río Magdalena y sus afluentes (1).

Entre 2008 y 2019 se notificaron 267 casos confirmados de LV en el país, provenientes de los departamentos de Bolívar, Sucre, Córdoba, Huila, Tolima, Santander y Cundinamarca. Aunque estas cifras demuestran que existe un carácter focal, esporádicamente se presentan casos nuevos en áreas que antes se consideraban libres de la enfermedad, incluyendo algunos núcleos urbanos del departamento de Sucre y de Bolívar (2-8).

En 2015, según el Sistema Nacional de Vigilancia en Salud Pública (SIVIGILA) en el departamento de Bolívar se registraron 14 casos de LV, de estos 13 ocurrieron en El Carmen de Bolívar y uno en San Juan Nepomuceno, específicamente en la vereda Toro del Corregimiento de San Cayetano, donde no había registros epidemiológicos de esta forma clínica. El caso que dio origen a este estudio, fue de un menor de sexo masculino, con un año de edad con cuadro clínico compatible con la enfermedad y diagnóstico confirmado, el cual fue notificado en la semana epidemiológica 13 de 2015 (9).

Estudios previos de la LV en el Caribe colombiano han mostrado que Lutzomyia evansi (NuñezTovar, 1924) es el vector principal de Leishmania infantum Nicolle, 1908, agente etiológico de esta forma clínica de la enfermedad (10). La transmisión usualmente sucede en el ambiente domiciliar, donde están presentes los caninos, que en la región han sido señalados como los principales reservorios de estos parásitos $(11,12)$. Los trabajos de identificación de preferencias alimenticias realizados en San Andrés de Sotavento indican que Lu. evansi, se alimenta con mayor frecuencia de Homo sapiens sapiens que de Canis familiaris y Didelphis marsupialis (13), así mismo, en el municipio de Ovejas, en estudios de determinación de fuentes de ingesta se encontró que esta especie se alimenta de Equus asinus (25\%), Bos taurus indicus (22,5\%), Homo sapiens sapiens $(16,25 \%)$, Equus caballus (5\%), Sus scrofa $(5 \%)$, Tamandua mexicana $(2,5 \%)$, Mabuya sp. (2,5\%), Proechimys guyanensis $(2,5 \%)$ y Gallus gallus $(1,25 \%)(14)$.

Teniendo en cuenta el carácter focal de la leishmaniasis y el registro de LV en una vereda de San Juan Nepomuceno, sin antecedentes de la enfermedad, este trabajo tuvo como objetivo caracterizar la fauna flebotomínea asociada al caso, sus porcentajes de infección con Leishmania spp., la identidad de los animales que sirven como fuente de ingesta y el diagnóstico serológico de leishmaniasis canina (LCa).

\section{MÉTODOS}

Diseño del estudio: El estudio se puede catalogar como transversal descriptivo

Área de estudio: El estudio fue desarrollado en área rural del departamento de Bolívar, en la vereda Toro del corregimiento de San Cayetano, municipio de San Juan de Nepomuceno, el cual hace parte del corredor ecológico de Los Montes de María, que constituye el foco más importante de leishmaniasis del Caribe colombiano. Este corregimiento, donde no se habían 
registrado casos autóctonos de leishmaniasis visceral hasta el año 2015, tiene una población de 12382 habitantes. Geográficamente, el corregimiento está ubicado a $10^{\circ} 08^{\prime} \mathrm{N}$, y $75^{\circ} 14^{\prime} \mathrm{W}$. En el municipio se registra una temperatura media anual de $27,7^{\circ} \mathrm{C}$ y una precipitación promedio que varía entre 800 y $1500 \mathrm{~mm}$ por año, la zona de vida corresponde a bosque seco tropical según Holdridge en 1967.

Muestreo de flebotomíneos: Se realizó un muestreo puntual de insectos con cuatro trampas CDC de luz emitidas por diodos, descritas en detalle por Castillo P, et.al (15). Tres trampas se instalaron en el peridomicilio, hasta $100 \mathrm{~m}$ de la vivienda, distribuidas en un corral de cerdos, corral de bovinos y área de pastoreo. Otra trampa se ubicó en el extradomicilio, en un relicto de bosque a más de $100 \mathrm{~m}$ de la vivienda. Las trampas fueron operadas entre las 18:00 y las 06:00 horas, durante los días 5 y 6 de mayo de 2015. Para la determinación taxonómica de cada flebotomíneo se separó la cabeza, el ala y los últimos segmentos del abdomen, que fueron clarificados en ácido láctico y fenol (en proporción 1:1). La determinación de especie se realizó con las claves taxonómicas de Young y Duncan (1994) y Galati (2015). Con base en el número absoluto y porcentual de cada especie flebotomínea determinada se estimó la riqueza y abundancia en el área de estudio.

Detección de ADN de parásitos del género Leishmania que infectan flebotomíneos: Los flebotomíneos hembra, sin evidencias de ingesta sanguínea, fueron seleccionados para la detección de infección natural con Leishmania spp. y organizados en grupos conformados por 1 a 20 individuos de la misma especie y trampa. A partir de estos se extrajo el ADN mediante un protocolo de altas concentraciones de sales descrito previamente por (16). Para detectar la presencia de ADN de parásitos del género Leishmania se realizó una PCR convencional con los cebadores 13A (5'-GTG GGG GAG GGG CGT TCT-3') y 13B (5'-ATT TTA CAC CAA CCC CCA GTT-3') (17), los cuales delimitan un fragmento de 120 pares de bases (pb) de la región conservada del minicírculo del kinetoplasto. La PCR se desarrolló en un volumen de $25 \mu \mathrm{l}$ con la enzima Platinum ${ }^{\circledR}$ Taq DNA Polymerase, según las recomendaciones del fabricante. Como control positivo se utilizó ADN de una cepa de referencia de Leishmania braziliensis y como control negativo, grupos de machos y agua ultrapura.

Para tipificar la especie de Leishmania infectante se realizó una PCR semi-anidada, usando como blanco la región del espaciador transcrito interno dos (ITS-2), ubicada entre los genes rRNA 5.8S y rRNA LSU. En la primera reacción se usaron los cebadores LITSV (5'ACACTCAGGTCTGTAAAC-3') y LITSR (5'-CTGGATCATTTTCCGATG-3', que flanquean una secuencia de 1088 pb (18), mientras que en la segunda reacción se emplearon los cebadores LITSV y L5.8SR (5'-AAGTGCGATAAGTGGTA-3'), los cuales delimitan un segmento de 750 $\mathrm{pb}(18,19)$. Ambas reacciones se realizaron en un volumen total de $25 \mu \mathrm{l}$, que incluyó $3 \mu \mathrm{l}$ de la solución con el ADN extraído o una dilución (1/50) de los productos de PCR de la primera reacción. Las condiciones de la mezcla de PCR correspondieron a las antes descritas, al igual que los controles positivo y negativo usados en la primera reacción, mientras que para la segunda reacción de PCR se incluyó además, el control positivo y negativo del producto de la PCR de la primera reacción. Los amplicones fueron analizados mediante electroforesis en gel de agarosa y aquellos que presentaron el tamaño esperado y mostraron una concentración de ADN superior a $100 \mathrm{ng} / \mathrm{ul}$ fueron secuenciados a través de la estrategia de Sanger (20), incluida la cepa del parásito usada como control positivo. Con las secuencias obtenidas del estudio y las secuencias del material tipo depositadas en Gen Bank, se realizó un alineamiento múltiple 
utilizando el programa MAFFT (21) incluido en el programa Geneious V. 8.0; en el programa Jmodel test (22) se cargó la matriz de secuencias alineadas para estimar el modelo de sustitución que mejor describe la variación observada en el grupo de datos. La reconstrucción del árbol filogenético se realizó con el programa MrBayes 3.2.6 (23) con cuatro Cadenas de Markov Monte Carlo corridas simultáneamente por 1100000 generaciones, muestreadas cada 200 generaciones y burn-in de 1.375 .

La frecuencia mínima de infección se estimó como la presencia de al menos un insecto positivo en cada grupo de flebotomíneos que presentó el amplicón esperado. El número mínimo de flebotomíneos infectados se calculó teniendo como base el total de individuos de cada especie

Identificación de vertebrados usados por los flebotomíneos para la ingesta sanguínea: Los flebotomíneos que se encontraron alimentados con sangre fueron procesados mediante una PCR múltiple del gen Citocromo b (Cyt B), que detecta cinco especies de vertebrados correspondientes a Bos indicus, Sus scrofa, Capra hircus, Canis familiaris y Homo sapiens sapiens (Linnaeus, 1758). Para la PCR se usó una pareja de cebadores universales y se adicionó un cebador delantero específico para cada especie de vertebrado, cuyo tamaño y secuencia fueron descritos en detalle por (24). La enzima ExAct Taq DNA Polymerase ${ }^{\mathrm{TM}}$ (M. Biotech) fue seleccionada para la PCR, en un volumen de mezcla de $25 \mu$, según las condiciones recomendadas por el fabricante. La temperatura de alineamiento se calculó con la herramienta Oligoanalyzer 3.1 (https://eu.idtdna.com/calc/analyzer) y el perfil térmico resultante consistió de una activación inicial a $95^{\circ} \mathrm{C}$ por 2 minutos, seguida de 35 ciclos de amplificación, compuestos de una etapa de desnaturalización a $95^{\circ} \mathrm{C}$ por 20 segundos, alineamiento de cebadores a $58^{\circ} \mathrm{C}$ por 40 segundos, extensión a $72^{\circ} \mathrm{C}$ por 35 segundos y una extensión final a $72^{\circ} \mathrm{C}$ durante 5 minutos. Como control positivo se utilizó ADN extraído de sangre periférica o hígado de B. indicus, S. scrofa, C. hircus, C. familiaris y H. sapiens.

Los productos amplificados fueron separados mediante electroforesis en gel de agarosa al $2 \%$ por 45 minutos, a 80 voltios, la tinción del ADN se realizó con GelStar® 0.5X. El tamaño de los amplificados obtenidos se determinó con un marcador de peso molecular de 100bp (Promega ${ }^{\mathrm{TM}}$ ).

Con base en los resultados de identificación sanguínea, se estimó la frecuencia de uso de cada fuente alimenticia por los flebotomíneos.

Seroprevalencia de anticuerpos de tipo IgG anti-Leishmania en caninos: A los caninos presentes en la vivienda donde se presentó el caso de LV, se les tomó una muestra de sangre periférica por punción de la vena cefálica, después de haber obtenido el consentimiento informado verbal del jefe del hogar. De cada muestra se obtuvo el suero sanguíneo que fue usado para la detección de anticuerpos de tipo IgG-anti Leishmania spp. mediante inmunofluorescencia indirecta (IFI). Esto se realizó según el protocolo descrito por (25). Como antígenos se emplearon promastigotes de una cepa de Le. braziliensis aislada de un paciente del municipio de Ovejas (MHOM/CO/2014/77) y como conjugado se usó anti-IgG de perro marcada con fluoresceína (Sigma-Aldrich). Los resultados de la IFI se interpretaron con base en lo recomendado por la Guía protocolo para la vigilancia en Salud Pública de leishmaniasis del Instituto Nacional de Salud de Colombia (2), según la cual se considera que un animal es positivo cuando se obtienen lecturas de fluorescencia a una dilución del suero igual o mayor a 1/32. 
Aspectos éticos: Todos los procedimientos se realizaron teniendo en cuenta las leyes y normas colombianas para toma de muestras en los caninos, los procedimientos se realizaron de acuerdo con la Ley 84 de 1989. La recolección de los flebotomíneos está amparada en el permiso marco de colecta otorgado por la ANLA a la Universidad de Sucre, incluido el grupo de Investigaciones Biomédicas, identificado con la resolución 0391 de abril del 2015.

\section{RESULTADOS}

\section{Riqueza y abundancia de flebotomíneos del género Lutzomyia}

Se recolectaron 2178 flebotomíneos hembra del género Lutzomyia, con una riqueza de tres especies correspondientes a Lutzomyia evansi (Nunez-Tovar, 1924), Lu. dubitans Sherlock, 1962) y Lu. trinidadensis (Newstead, 1922). Lutzomyia evansi fue la especie más abundante con el $99,60 \%(n=2169 / 2178)$, seguida de Lu. dubitans $0,30 \%(n=6 / 2178)$ y Lu. trinidadensis $0,1 \%(n=3 / 2178)$. La mayor abundancia se registró en el área usada para el pastoreo de ganado vacuno donde se atrapó el 53,5 \% $(n=1165 / 2178)$ de los flebotomíneos, seguida por el corral de bovinos con el $21,4 \%(n=467 / 2178)$, corral de cerdos con el $17,8 \%(n=388 / 2178)$ y el relicto de bosque con el $7,3 \%(n=158 / 2178)$. De 2178 flebotomíneos recolectados, 1101 se encontraron alimentados con sangre y en 1077 no se observó ingesta sanguínea

\section{Detección de ADN de parásitos del género Leishmania que infectan flebotomíneos.}

Se evaluaron 61 grupos de flebotomíneos conformados por 1077 hembras del género Lutzomyia que no presentaron restos sanguíneos. Mediante la PCR de la región conservada del minicírculo se amplificó el fragmento esperado de 120 pb en 29/1070 grupos de Lu. evansi y 1/4 individuos de Lu. dubitans. Los flebotomíneos de los grupos positivos fueron capturados en el relicto de bosque y en el área de pastoreo. Por otra parte, con la PCR semi-anidada de la región del ITS2 se amplificó el fragmento esperado de 750 pb en 2/1070 grupos de Lu. evansi atrapados en el área de pastoreo.

De 30 grupos de hembras del género Lutzomyia, en los que se amplificó la región conservada del minicírculo, ocho muestras exhibieron valores de concentración de ADN superiores a $100 \mathrm{ng} / \mathrm{ul}$. A partir de estos productos de PCR se obtuvieron tres secuencias nucleotídicas consenso de 118 pb, las cuales fueron alineadas con secuencias homólogas de especies del mismo género. Con BLAST se determinó que la primera y la última posición de las secuencias obtenidas son homólogas a la región comprendida entre las posiciones 16 y 703 del minicírculo de Le. infantum (número de acceso AF103739), segmento que corresponde a la región conservada, utilizada en la discriminación de tripanomátideos. Se observaron valores de cobertura del $100 \%$, identidad del $99 \%$ y un valor E de $2 \mathrm{e}-180$. En el árbol filogenético se evidencia que las secuencias LeCBSCVTorG4, LeCBSCVTorG8 y LeCBSCVTorG34 detectadas en $L u$. evansi se agrupan, con un alto valor de soporte de rama, con especies del complejo Le. donovani (fig.2), cuyo único representante en América es Le. infantum. Esto permite inferir que las tres secuencias nucleotídicas detectadas en $L u$. evansi pertenecen a esta especie de parásito, lo que corresponde a una frecuencia mínima de infección del 0,003\% (3/1070).

Por otro lado, de uno de los dos grupos de Lu. evansi en los que se amplificó y secuenció la región ITS2, se obtuvo una secuencia consenso de $761 \mathrm{pb}$ que fue alineada con secuencias homólogas de especies del género Leishmania. El análisis en BLAST arrojó que ésta es homóloga al segmento comprendido entre la posición 3925 y 4435 de Le. braziliensis (número de acceso AOSE02000231.1). La topología del árbol y los valores de soporte de rama indican que la secuencia (LeCBSJScVtG45) detectada en Lu. evansi corresponde a Le. braziliensis (datos no mostrados), lo que equivale a una frecuencia mínima de infección del 0,0009\% (1/1070). 
Identificación de vertebrados usados por los flebotomíneos como fuente de ingesta sanguínea.

La PCR múltiple del gen mitocondrial citocromo b se aplicó al 44,50\% (N: 490/1101) de las hembras que presentaron restos macroscópicos de sangre en el tracto digestivo. En el 78,76\% (N: 386/490) de las muestras procesadas se obtuvo por lo menos un amplicón del tamaño esperado. La identificación arrojó que el 16,73\% (N: 82/490) de las hembras de Lu. evansi ingurgitadas y evaluadas se alimentaron de H. sapiens, el 16,32\% (N: 80/490) de C. hircus, el 12,45\% (N: 61/490) de S. scrofa, el 11,63 \% (N: $57 / 490$ ) de B. indicus y el 9,79\% (N: 48/490) de C. familiaris. También se detectó que el 11,84\% (N: $58 / 490$ ) de las hembras de Lu. evansi presentaron ingestas mixtas de animales (Tabla I), lo que indica que el $78,76 \%$ (N: 386/490) de las muestras procesadas tenían rastros de sangre Seroprevalencia de anticuerpos de tipo $\lg G$ anti-Leishmania en caninos

Uno de los ocho caninos analizados por serología presentó anticuerpos de tipo lgG frente a Leishmania spp. cuando se evaluó su suero sanguíneo a una dilución de $1 / 32$, por lo cual fue diagnosticado con leishmaniasis canina.

Figura 1. Área rural del corregimiento de San Cayetano, municipio de San Juan Nepomuceno, Bolívar, Colombia. Fuente: QGIS v. 2.18.14 para macOS Sierra.

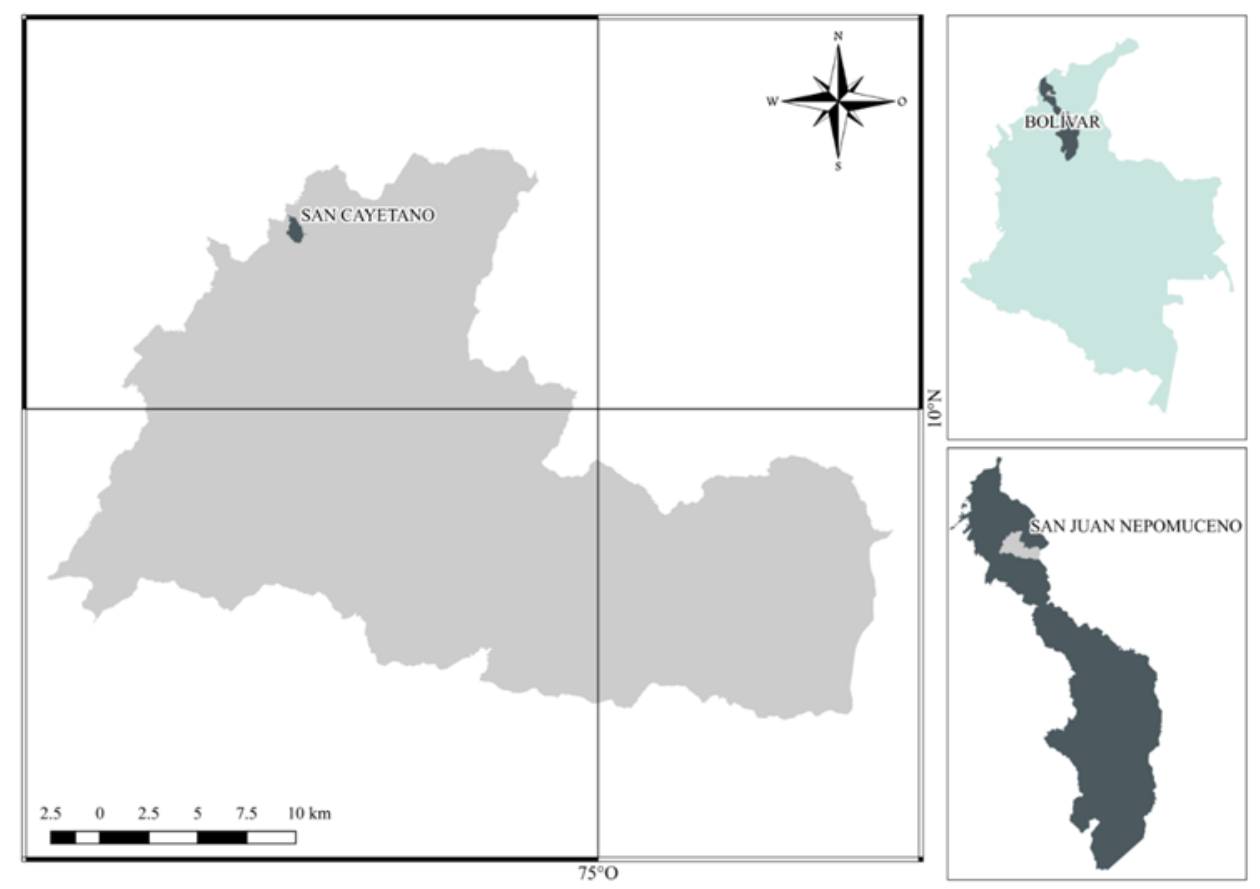

Fuente: Elaboración propia. 
Figura 2. Análisis filogenético de la región conservada del minicírculo de especies del género Leishmania

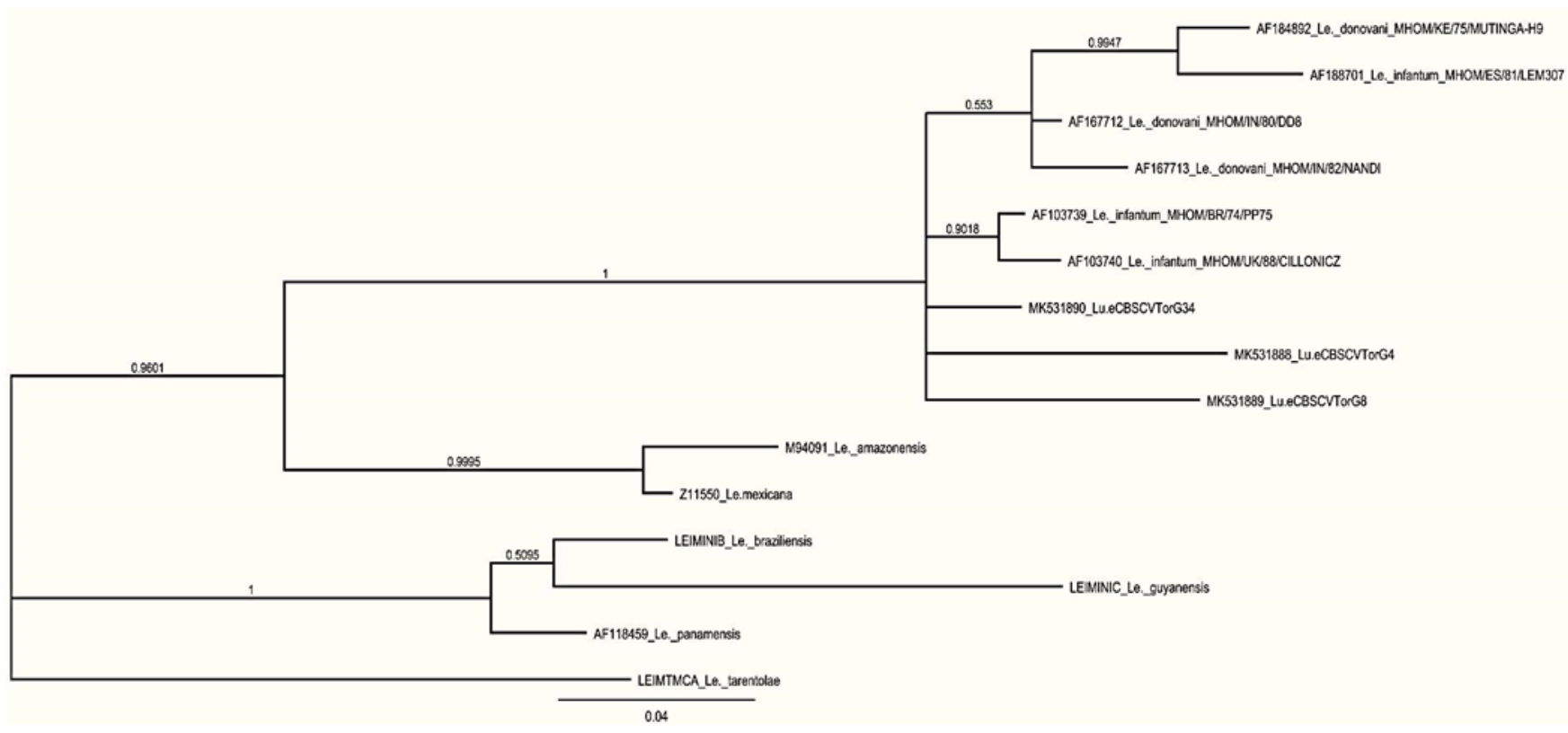

Fuente: Elaboración propia

\section{DISCUSIÓN}

En la incriminación de una especie de flebotomíneo como vector de Leishmania spp. es necesario demostrar que su abundancia temporal se solapa con los casos de la enfermedad, que tiene capacidad para infectarse y transmitir los parásitos, y que existe vínculo epidemiológico entre parásitos, humanos y vertebrados reservorios $(27,28)$. Los resultados del presente trabajo indican que Lu. evansi cumple la mayoría de estos criterios y por tanto podría actuar como vector de Le. infantum y Le. braziliensis en la vereda Toro. El estudio realizado demuestra no solo la asociación de Lu. evansi con el caso de LV, sino también que ésta es la especie de flebotomíneo más abundante en el ambiente peridoméstico, que se infecta con los parásitos causantes de la enfermedad y que ostenta un comportamiento ecléctico de picadura. Esto último permite probar el vínculo epidemiológico entre el humano y los potenciales reservorios, que probablemente incluyen a los caninos, puesto que se encontró un perro cuyo diagnóstico serológico arrojó positivo para leishmaniasis canina.

En primera instancia, desde el punto de vista entomológico es importante destacar el hallazgo de Lu. evansi, Lu. dubitans y Lu. trinidadensis, que corresponde al primer registro de estas especies en el municipio de San Juan Nepomuceno. Lutzomyia evansi fue la especie predominante en el área, lo cual es consistente con resultados de trabajos previos en Los Montes de María, donde la presencia de esta especie, en alta abundancia, $(29,30)$ ha sido asociada a brotes de LV en los departamentos de Sucre y Bolívar, como en el foco de El Hobo, El Carmen de Bolívar (3) y en Cartagena (31). Se ha planteado que la amplia distribución de 
esta especie y su abundancia en el domicilio se debe a su capacidad para adaptarse a los ambientes modificados por el humano (32).

Tabla 1. Frecuencia de ingestas sanguíneas mixtas en hembra de Lutzomyia evansi recolectadas en la vereda Toro, San Juan Nepomuceno, Bolívar.

\begin{tabular}{|c|c|c|}
\hline Especie flebotominea & $\begin{array}{l}\text { Combinaciones de } \\
\text { Ingesta alimenticia }\end{array}$ & Frecuencia (n/n) \% \\
\hline Lu. evansi & B. indicus + C. hircus & $1,63(8 / 490)$ \\
\hline Lu. evansi & B. indicus $+H$. sapiens. & $1,42(7 / 490)$ \\
\hline Lu. evansi & B. indicus + S. scrofa & $0,40(2 / 490)$ \\
\hline Lu. evansi & C. familiaris + S. scrofa & $0,81(4 / 490)$ \\
\hline Lu. evansi & C. familiaris + C. hircus & $0,61(3 / 490)$ \\
\hline Lu. evansi & C. familiaris $+H$. sapiens & $1,02(5 / 490)$ \\
\hline Lu. evansi & C. familiaris $+B$. indicus & $0,20(1 / 490)$ \\
\hline Lu. evansi & S. scrofa $+H$. sapiens & $2,44(12 / 490)$ \\
\hline Lu. evansi & S. scrofa + C. hircus & $0,61(3 / 490)$ \\
\hline Lu. evansi & H. sapiens + C. hircus & $1,22(6 / 490)$ \\
\hline Lu. evansi & $\begin{array}{c}\text { B. indicus }+H . \text { sapiens }+C . \\
\text { hircus }\end{array}$ & $0,81(4 / 490)$ \\
\hline Lu. evansi & $\begin{array}{c}\text { B. indicus }+S . \text { scrofa }+C . \\
\text { hircus }\end{array}$ & $0,20(1 / 490)$ \\
\hline Lu. evansi & $\begin{array}{c}\text { C. familiaris }+H . \text { sapiens }+ \\
\text { C. hircus }\end{array}$ & $0,40(2 / 490)$ \\
\hline
\end{tabular}

Fuente: Elaboración propia

En segundo lugar, la detección del material genético (ADN) de Le. infantum en el 0,003\% ( $\mathrm{N}: 3 / 1070)$ de las hembras de Lu. evansi, concuerda con los antecedentes de infección natural con este parásito registrados en el departamento de Córdoba (33), donde ha sido señalada como vector de Le. infantum, con frecuencias de infección que fluctuan entre $0,1 \%$ y $1,6 \%$ en los municipios de San Andrés de Sotavento (34) y Sahagún $(34,35)$. Así mismo en el municipio de El Carmen de Bolívar, departamento de Bolívar, en el cual la frecuencia de infección es de $2,71 \% \underline{(36)}$.

Por otra parte, el hallazgo durante el presente estudio de Le. braziliensis en Lu. evansi con una frecuencia mínima de infección del 0,0009\% (1/1070), es consistente con lo antes observado en El Carmen de Bolívar, Bolívar, donde la frecuencia de infección fue del 0,07\% $\underline{(36)}$. 
Similarmente, investigaciones realizadas en el municipio de Ovejas, Sucre, han arrojado en este flebotomíneo una frecuencia de infección del $0,12 \%$ con parásitos del complejo Le. (Viannia) braziliensis (37), a lo que se adiciona el aislamiento y la tipificación de una cepa de Le. braziliensis a partir de una hembra de Lu. evansi capturada en el municipio de Colosó, Sucre (38). Estos datos en conjunto indican que la población de Lu. evansi evaluada en el presente estudio presenta un comportamiento similar al observado en otros focos de leishmaniasis del Caribe colombiano, por lo tanto, su abundancia y la frecuencia de infección hallada serían suficientes para mantener el ciclo epidemiológico de la leishmaniasis y para justificar la ocurrencia de casos humanos de la enfermedad.

En cuanto al vínculo epidemiológico entre Le. infantum, Lu. evansi y los potenciales reservorios, el estudio de las fuentes de ingesta sanguínea confirma que Lu. evansi es antropofágica, lo que se corresponde con trabajos previos con cebo humano protegido, trampas de atracción y estudios moleculares de fuentes de ingesta $\underline{(5,}, \underline{13}, \underline{14}, \underline{35})$. Este hecho puede explicarse por la presencia de alcoholes primarios como el 1-octen-3-ol presentes en la respiración humana, que sirven como atrayentes para los flebotomíneos (39), y por la disponibilidad del humano en el domicilio.

Es de resaltar que C. familiaris fue usado por Lu. evansi como fuente de ingesta sanguínea, hallazgo que contrasta con los resultados de un estudio realizado en el municipio de Ovejas, Sucre, en el cual no se detectó sangre de este animal en el tracto digestivo de hembras alimentadas. Es probable que estas diferencias se deban a las técnicas de caracterización usadas, pues, si bien es cierto que la secuenciación ofrece resultados precisos de identificación taxonómica, también desestima la proporción de ingestas mixtas cuando no se clonan los amplicones que presentan secuencias con productos mezclados. En términos de la bionomía del insecto, la frecuencia de uso de fuentes de ingestas indica que el vector presenta un comportamiento ecléctico de picadura puesto que se halló una importante proporción de ingestas mixtas (C. familiares, C. hircus, S. scrofa, B. indicus y H. sapiens). Esto indica que Lu. evansi es capaz de alimentarse de varias especies de vertebrados antes de completar su ingesta sanguínea $(40,41)$, y durante este proceso, aumenta la probabilidad de tener contacto con animales infectados con parásitos del género Leishmania, como el canino hallado en la vereda Toro. Esto sumado a los antecedentes epidemiológicos del insecto y de los caninos, indican que probablemente ambas especies facilitan la transmisión, circulación y el mantenimiento de los parásitos causantes de leishmaniasis en la zona de estudio.

Considerando la abundancia que exhibe Lu. evansi se infiere que su presencia constituye un riesgo potencial para las poblaciones humanas que habitan en la vereda Toro, puesto que no solo se infecta con parásitos causantes de LV, sino también porque sus hábitos hematófagos le permiten entrar en contacto con hospederos de Leishmania spp. como el perro doméstico. Aunque se desconoce si los otros vertebrados utilizados como fuente de ingesta también pueden servir como hospederos de Leishmania spp., el conocimiento de la frecuencia de uso de cada especie de vertebrado ofrece una oportunidad para el diseño, evaluación e implementación de medidas de control vectorial de la enfermedad. 


\section{CONCLUSIONES}

Lutzomyia evansi es la especie de flebotomíneo más abundante en la vereda Toro, San Juan Nepomuceno, Bolívar. Esta especie flebotomínea se infecta con Le. infantum y Le. braziliensis en frecuencias lo suficientemente altas para mantener la transmisión y soportar la aparición del caso humano de la enfermedad. Así mismo, presenta un comportamiento de picadura ecléctico que permite el vínculo entre los caninos infectados con el parásito y los humanos. Teniendo en cuenta la amplia distribución de esta especie en el Caribe colombiano y los fenómenos de cambio social que a su vez modelan los cambios en la epidemiología de la leishmaniasis, la presencia de Lu. evansi podría ser un factor determinante en la aparición de nuevos casos de la enfermedad en la región.

Contribución de los autores: "Conceptualización, A.J.P.D, E.E.B.M y L.C.A.; metodología, todos los autores.; software, M.A.A.S y A.J.P.D.; curación de datos: M.A.A.S y L.M.M.P.; análisis formal, M.A.A.S, L.M.M.P,M.E.R.R, A.J.P.D y E.E.B.M.; investigación, M.A.A.S, L.M.M.P,M.E.R.R, L.C.A y A.J.P.D.; visualización, M.A.A.S, L.M.M.P y M.E.R.R.; escritura: preparación del borrador original, M.A.A.S y L.M.M.P.; escritura: revisión y edición, todos los autores; supervisión, A.J.P.D y E.E.B.M.; administración del proyecto A.J.P.D y E.E.B.M. Todos los autores han leído y aceptado la versión publicada del manuscrito".

Fondos: Esta investigación no recibió fondos externos.

Agradecimientos: A la Secretaria de Salud Departamental de Bolívar, a mis colegas Luis Roberto Romero Ricardo, Natalia Lastre Meza, José Gabriel Vergara Meza y Jorge Luis Rodríguez Jiménez.

Conflictos de intereses: Los autores declaran no tener ningún conflicto de intereses.

\section{REFERENCIAS}

1. Fernández J, Charry T, Bello F, Escovar J, Lozano C, Ayala M, et al. Prevalencia de leishmaniasis visceral canina en municipios de Huila-Colombia. Acta biol Colomb. 2002; 4(3):

278-285.

https://revistas.unal.edu.co/index.php/revsaludpublica/article/view/18534/19446.

2. Bejarano E, Uribe S, Rojas W, Darío I. Phlebotomine sand flies (Diptera: Psychodidae) associated with the appearance of urban leishmaniasis in the city of Sincelejo, Colombia. Mem Inst Oswaldo Cruz. 2002; 97(5): 645-647. Doi: http://dx.doi.org/10.1590/S007402762002000500010 . https://www.scielo.br/scielo.php?pid=S007402762002000500010 \&script=sci arttext.

3. Cortés L. Leishmaniasis transmission focus in El Hobo, Carmen de Bolívar, Bolívar, Colombia. Biomédica. 2006; $26 \quad$ Suppl 1: 236-241. https://revistabiomedica.org/index.php/biomedica/article/view/1518/1649.

4. Cortés L, Fernández J. Especies de Lutzomyia en un foco urbano de leishmaniasis visceral y cutánea en El Carmen de Bolívar, Bolívar, Colombia. Biomédica. 2008; 28(3): 433-440. Doi: https://doi.org/10.7705/biomedica.v28i3.81. http://www.scielo.org.co/scielo.php?pid=S0120$41572008000300013 \&$ script=sci arttext\&tlng=pt. 
5. Cortes L, Pérez-Doria, Bejarano E. Flebotomíneos (Diptera: Psychodidae) antropofílicos de importancia en salud pública en Los Montes de María, Colombia. Rev Cubana Med Trop. 2009; 61(3): 6. http://scielo.sld.cu/scielo.php?script=sci arttext\&pid=S037507602009000300003.

6. Cortés L, Pereira R. Evaluación ecoepidemiológica de la leishmaniasis en El Carmen de Bolívar. Biomédica. 2011; 31(95): 95.

7. Fuya P, Pisciotti I, Pérez S, Lugo L, Ferro C. Presencia de Lutzomyia evansi (NúñezTovar, 1924) vector de Leishmania chagasi en área urbana de Cartagena, Colombia. Biomédica. 2011; 31(3): 111-112.

8. Zambrano-Hernandez P, Ayala-Sotelo M, Fuya-Oviedo P, Montenegro-Puentes C, AyaVanegas N, Aguilera-Jaramillo G, et al. Brote urbano de leishmaniasis visceral en Neiva, Colombia. Revista de Salud Pública. 2015; 17:514-527. https://www.scielosp.org/article/rsap/2015.v17n4/514-527/.

9. SIVIGILA. (2016). Sistema Nacional de Vigilancia en Salud Pública. Información epidemiológica. Informe de eventos. https://www.ins.gov.co/buscadoreventos/Informesdeevento/Leishmaniasis\%202016.pdf.

10. Travi B, Vélez I, Brutus L, Segura I, Jaramillo C, Montoya J. Lutzomyia evansi, an [alternative] vector of Leishmania chagasi in a Colombian focus of visceral leishmaniasis. Trans Royal Soc Trop Med Hyg.1990; 84(5): 676-677. Doi: 10.1016/00359203(90)90142-2. https://pubmed.ncbi.nlm.nih.gov/2278068/.

11. Rivero-Rodríguez M. Identificación de Leishmania chagasi en canis familiaris en un foco de los Montes de María. Universidad de Sucre. 2003; Tesis. 2003. https://repositorio.unisucre.edu.co/bitstream/001/50/2/T616.9364007\%20R621.pdf.

12. Rivero-Rodríguez M, Rodríguez-Jiménez J, Pérez-Doria A, Bejarano E. Aislamiento de Leishmania infantum a partir de Canis familiaris en área urbana del Caribe Colombiano. Rev investig vet Perú. 2018; 29(3): 923-930. Doi: http://dx.doi.org/10.15381/rivep.v29i3.13708. http://www.scielo.org.pe/scielo.php?script=sci arttext\&pid=S160991172018000300023.

13. Montoya-Lerma J, Lane R. Factors affecting host preference of Lutzomyia evansi (Diptera: Psychodidae), a vector of visceral leishmaniasis in Colombia. Bulletin of Entomological Research. 1996; 86(01): 43-50. Doi: 10.1017/S0007485300052184. https://www.cambridge.org/core/journals/bulletin-of-entomologicalresearch/article/abs/factors-affecting-host-preference-of-Iutzomyia-evansi-dipterapsychodidae-a-vector-of-visceral-leishmaniasis-incolombia/7DAA65B81B1A4E786F2DD0E057A64506.

14. Paternina L, Verbel-Vergara D, Romero-Ricardo L, Pérez-Doria A, Paternina-Gómez M, Martínez L, et al. Evidence for anthropophily in five species of phlebotomine sand flies (Diptera: Psychodidae) from northern Colombia, revealed by molecular identification of bloodmeals. Acta trop. 2016; 153: 86-92. Doi: 10.1016/j.actatropica.2015.10.005. https://www.sciencedirect.com/science/article/abs/pii/S0001706X15301285.

15. Castillo P, Bejarano E, Pérez-Doria A. Validación de las trampas CDC con Diodos Emisores de Luz (LED) en la captura de insectos flebotomíneos (Diptera: Psychodidae). Universidad De Sucre. 2011; Tesis.

16. Pérez-Doria A, Romero-Ricardo L, Bejarano E. Cambiando la forma de extraer el ADN en insectos vectores (Diptera: Psychodidae) de Leishmania spp. Memorias del XXXV Congreso de la Sociedad Colombiana de Entomología. 2008. 
17. Rodgers M, Popper S, Wirth D. Amplification of kinetoplast DNA as a tool in the detection and diagnosis of Leishmania. Exp Parasitol; 1990. 71(3): 267-275. Doi: https://doi.org/10.1016/0014-4894(90)90031-7. https://www.sciencedirect.com/science/article/abs/pii/0014489490900317.

18. El Tai N, Osman O, El Fari M, Presber W, Schönian G. Genetic heterogeneity of ribosomal internal transcribed spacer in clinical samples of Leishmania donovanispotted on filter paper as revealed by single-strand conformation polymorphisms and sequencing. Trans Royal Soc Trop Med Hyg. 2000. 94(5): 575-579. Doi: https://doi.org/10.1016/S0035-9203(00)90093-2. https://www.sciencedirect.com/science/article/abs/pii/S0035920300900932.

19. De Almeida M, Steurer F, Koru O, Herwaldt B, Pieniazek N, da Silva A. Identification of Leishmania spp. by molecular amplification and DNA sequencing analysis of a fragment of the rRNA internal transcribed spacer 2. J Clin Microbiol. 2011; 49(9):3143-9. Doi: 10.1128/JCM.01177-11.

20. Sanger F. DNA sequencing with chain-terminating inhibitors. Proc. Natl. Acad. Sci. USA. 1983; 74(12):5463-7.Doi: https://www.pnas.org/content/74/12/5463. 10.1073/pnas.74.12.5463.

21. Katoh K, Standley D. MAFFT multiple sequence alignment software version 7: improvements in performance and usability. Mol Biol Evol. 2013; 30(4): 772-780. Doi:10.1093/molbev/mst010. https://academic.oup.com/bioinformatics/article/26/15/1899/188098?login=true.

22. Darriba D, Taboada G, Doallo R, Posada D. jModelTest 2: more models, new heuristics and parallel computing. Nat Methods. 2012; 9(8): 772. Doi:10.1038/nmeth.2109. https://pubmed.ncbi.nlm.nih.gov/22847109/.

23. Huelsenbeck J, Ronquist F. MRBAYES: Bayesian inference of phylogenetic trees. Bioinformatics. 2001; 17(8): 754-755.

Doi: https://doi.org/10.1093/bioinformatics/17.8.754. https://pubmed.ncbi.nlm.nih.gov/11524383/.

24. Kent $R$, Norris $D$. Identification of mammalian blood meals in mosquitoes by a multiplexed polymerase chain reaction targeting cytochrome B. Am J Trop Med Hyg. 2005; 73(2): 336-342. https://www.ajtmh.org/content/journals/10.4269/ajtmh.2005.73.336.

25. Camargo M. Fluorescent antibody test for the serodiagnosis of American trypanosomiasis. Technical modification employing preserved culture forms of Trypanosoma cruzi in a slide test. Rev Inst Med Trop Sao Paulo. 1966; 8(5): 227-234. https://pubmed.ncbi.nlm.nih.gov/4967348/.

26. Ministerio de la Protección Social y Salud. Guía protocolo para la vigilancia en Salud Pública de leishmaniasis Instituto Nacional de Salud de Colombia, Subdirección de Vigilancia y Control en Salud Pública, Protocolo de vigilancia y control de Leishmaniasis. 2010; PRO-R02.003.0000-016, 29.

27. Killick-Kendrick R. Studies and criteria for the incrimination of vectors and reservoir hosts of the leishmaniases. Proceedings of the International Workshop on Research on Control Strategies for the Leishmaniases. 1998; Ottawa, 1-4 June 280-282.

28. WHO. Control of the leishmaniasis: report of a meeting of the WHO Expert Committee on the Control of leishmaniases. WHO Technical Report Series, 949. 2010; Geneva, 2226 March. 
29. Pérez-Doria A, Oviedo E, Bejarano E. Lutzomyia (Diptera: Psychodidae) from the reserva Serranía de Coraza y Montes de María, Colombia. Rev. Colomb Entomol. 2008; 34(1): 98-101. http://www.scielo.org.co/scielo.php?script=sci arttext\&pid=S012004882008000100013.

30. Romero L, Lastre N, Pérez-Doria A, Bejarano E. Lutzomyia abonnenci and Lutzomyia olmeca (Diptera: Psychodidae), New Records for the Department. Acta biol.Colomb. 2013; 18 (2). http://www.scielo.org.co/scielo.php?script=sci arttext\&pid=S0120548X2013000200014.

31. Zambrano H, Ayala S, Fuya O, Barrazab O, Rodríguez T. Cartagena: nuevo foco de leishmaniasis visceral urbana en Colombia. Revista Ciencia en Desarrollo. 2016; 7(1): 83-91. http://www.scielo.org.co/pdf/cide/v7n1/v7n1a11.pdf.

32. Travi B, Adler G, Lozano M, Cadena H, Montoya-Lerma J. Impact of habitat degradation on phlebotominae (Diptera: Psychodidae) of tropical dry forests in Northern Colombia. J Med Entomol. 2002; 39(3): 451-456. Doi: 10.1603/0022-2585-39.3.451. https://academic.oup.com/ime/article-abstract/39/3/451/834931.

33. Montoya-Lerma J, Cadena H, Oviedo M, Ready PD, Barazarte R, Travi B. Comparative vectorial efficiency of Lutzomyia evansi and L. longipalpis for transmitting Leishmania chagasi. Acta Trop. 2003; 85:19-29. Disponible en: https://www.sciencedirect.com/science/article/pii/S0001706X02001894. https://www.sciencedirect.com/science/article/abs/pii/S0001706X02001894.

34. Travi B, Montoya J, Gallego J, Jaramillo C, Llano R, Velez I. Bionomics of Lutzomyia evansi (Diptera: Psychodidae) vector of visceral leishmaniasis in northern Columbia. $\mathrm{J}$ Med Entomol. 1996; 33(3): 278-285. Doi: 10.1093//medent/33.3.278. https://academic.oup.com/ime/article-abstract/33/3/278/892083.

35. Gonzalez C, Leon C, Paz A, Lopez M, Molina G, Toro D, Tovar C. Diversity patterns, Leishmania DNA detection, and bloodmeal identification of Phlebotominae sand flies in villages in northern Colombia. PLoS One. 2018; 13(1): e0190686. Doi:10.1371/journal.pone.0190686. https://journals.plos.org/plosone/article?id=10.1371/journal.pone.0190686.

36. Romero Ricardo L, Pérez-Doria A, Rodríguez J, Vergara J, Montesino A, Martínez L, Bejarano E. Lutzomyia evansi como vector permisivo de Leishmania braziliensis y Leishmania infantum en el departamento de Bolívar, Colombia. Biomédica. 2015; 35: 185.

37. Pérez-Doria, A. Búsqueda de infección natural con parásitos causantes de leishmaniasis cutánea en poblaciones flebotomíneas (Diptera: Psychodidae) en los Montes de María, Sucre, Colombia. Programa de maestría en microbiología, Universidad de Córdoba. 2011; Tesis.

38. Pérez-Doria A, Romero L, Martínez M, Rivero M, Bejarano E. Aislamiento y tipificación de una cepa de Leishmania braziliensis (Viannia) a partir de Lutzomyia evansi en el municipio de Colosó, Sucre. Biomédica. 2015; 35(Suplemento No. 4, XVI. Congreso Colombiano de Parasitología y Medicina Tropical), 112.

39. Hall D, Beevor P, Cork A, Nesbitt B, Vale G. 1-Octen-3-ol. A potent olfactory stimulant and attractant for tsetse isolated from cattle odours. International Journal of Tropical Insect Science. 1984; 5(5): 335-339. Doi: https://doi.org/10.1017/S1742758400008626. https://link.springer.com/article/10.1017/S1742758400008626.

40. Afonso M, Duarte R, Miranda J, Caranha L, Rangel E. Studies on the feeding habits of Lutzomyia (Lutzomyia longipalpis) (Lutz \& Neiva, 1912) (Diptera: Psychodidae: 
Phlebotominae) populations from endemic areas of American visceral leishmaniasis in northeastern Brazil. J Trop Med. 2012; 2012:858657. Doi: 10.1155/2012/858657. https://www.hindawi.com/journals/itm/2012/858657/.

41. Chiluiza Piedra P. Detección de la infección natural de Leishmania spp. En especímenes de Lutzomyia spp, y determinación molecular de los hospederos de los que se alimentan en las provincias de Esmeraldas y Chimborazo. Quito: Universidad de las Américas. 2016. http://dspace.udla.edu.ec/bitstream/33000/4860/1/UDLA-EC-TIB-2016-01.pdf. 